\title{
Relação entre a Persistência da Condução pela Via Lenta Nodal após Ablação de Taquicardia por Reentrada Nodal e a Recorrência Clínica
}

\author{
Márcio A. Silva, Maurício I. Scanavacca, André D’Avila, Ricardo Kuniyoshi, Eduardo A. Sosa \\ São Paulo, SP
}

Objetivo - Verificar se a persistência de salto nodal relaciona-se à taxa de recorrência de taquicardia por reentrada nodal (TRN) após ablação com radiofreqüência (RF) da via lenta do nó atrioventricular.

Métodos - Num seguimento de $20 \pm 12$ meses, foi analisada a recorrência de TRN em 126 pacientes consecutivos submetidos a ablação com $R F$ da via lenta nodal. $O$ critério de interrupção do procedimento foi a não reindução da TRN, após estimulação atrial programada, com e sem isoproterenol intravenoso. Ao final do procedimento, 98 pacientes não apresentavam salto nodal, e em 28 persistia o salto nodal elou o eco atrial.

Resultados -Houve recorrência clínica de TRN em 15 (11\%) pacientes: 9 no grupo sem salto nodal elou eco atrial e em 6 do grupo que persistiu com salto elou eco atrial. A recorrência tendeu a ser maior no $2^{\circ}$ grupo $(9 \%$ vs 21\%), mas não houve significância estatística entre os resultados $(p=0,09)$.

Conclusão-Desde que a TRN não possa ser induzida após a infusão de isoproterenol, a recorrência espontânea da arritmia após a ablação por $R F$ da via lenta nodal não é diferente entre pacientes que persistem ou não com salto nodal e/ou eco atrial.

Palavras-chave: taquicardia por reentrada nodal, ablação por cateter, via lenta

\section{Persistence of AV Conduction over the Slow Pathway after AVRNT Radiofrequency Ablation and its Relation to the Recurrences}

Purpose - The aim of this study is to verify whether the persistence of conduction over the slow pathway is related to an increased trend for recurrence.

Methods - Recurrence rate was retrospectively analyzed in 126 patients who underwent slow pathway radiofrequency $(R F)$ catheter ablation during a follow-up of $20 \pm 12$ months. The ablative procedure was interrupted when AVNRT was no longer induced by atrial stimulation after intravenous infusion of isoproterenol. Ninety-eight patients had no evidence of slow pathway whereas 28 patients persisted with AV node jump and atrial echo beat.

Results - There were 15 recurrences: $9 \%$ of those who had no evidence of slow pathway (9 of 98 patients) and $21 \%$ of those with $A V$ node jump and/or atrial echo beat but this difference was not statistically significant.

Conclusion - As long as AVNRT cannot be induced by atrial pacing and isoproterenol infusion after slow pathway RF catheter ablation, the presence of $A V$ node jump and/or atrial echo beat does not increase the risk of recurrence of AVNRT.

Key-words: atrioventricular reentrant tachycardia, catheter ablation, slow pathway
A ablação com radiofrequiência (RF) da via lenta do nó atrioventricular (AV) é um procedimento eficaz e seguro para tratamento definitivo da taquicardia por reentrada nodal (TRN). Entretanto, a recorrência espontânea da taquicardia tem sido relatada em até $14 \% \%^{1,9,12}$ dos pacientes

Instituto do Coração do Hospital das Clínicas da FMUSP

Correspondência: Eduardo Sosa - Incor - Av. Enéas C. Aguiar, 44 - 05403-000 São Paulo, SP

Recebido para publicação em 30/3/98

Aceito em 3/6/98 submetidos à ablação, podendo determinar a necessidade de um novo procedimento. Além disso, o bloqueio atrioventricular total (BAVT) inadvertido, a mais temida complicação deste procedimento, ocorre em até $3 \%{ }^{9}$ dos casos.

Em geral, a ablação da via lenta pode ser conseguida com poucos pulsos de $\mathrm{RF}^{12}$. Ocasionalmente, entretanto, $\mathrm{e}$, por razões não bem definidas, várias aplicações de RF são necessárias para eliminar o salto nodal. Por vezes, apesar da persistência do salto nodal e/ou do eco atrial, a arritmia não pode ser mais induzida, sugerindo que a modificação da via lenta nodal (persistência de salto nodal com ou sem eco 
atrial, sem indução de TRN) relaciona-se a uma baixa taxa de recorrência, mas o significado deste achado é ainda controverso ${ }^{1-5,7,12-14}$. Como aumentando o número de aplicações na tentativa de eliminar o salto nodal, a possibilidade de BAVT inadvertido também aumenta, o objetivo deste trabalho foi verificar se a taxa de recorrência relaciona-se mais com a adoção da modificação ou da ablação da via lenta nodal, como critério de fim do procedimento. Esta informação é relevante, pois, se a recorrência não for diferente em pacientes em que se obteve a modificação da via lenta, aplicações adicionais que objetivem eliminar o salto nodal podem ser evitadas, supostamente diminuindo o risco de BAVTinadvertido.

\section{Métodos}

Estudo retrospectivo, avaliando os resultados da ablação por cateter em 126 pacientes consecutivos, submetidos a ablação com RF da via lenta nodal, de outubro/92 a abril/96. A idade variou de cinco a 84 (média de 44) anos, sendo $98(78 \%)$ pacientes do sexo feminino.

O procedimento ablativo foi realizado no laboratório de eletrofisiologia após consentimento por escrito do paciente. As drogas antiarrítmicas foram suspensas, por no mínimo cinco meias-vidas. Os pacientes receberam $600 \mathrm{mg}$ de lincomicina intramuscular $1 \mathrm{~h}$ antes do procedimento, realizado sob sedação com midazolam e propofol intravenoso.

Introduzidos por punção da veia femoral direita e guiados por fluoroscopia, um cateter quadripolar 7Fr foi posicionado no átrio direito alto, para estimulação e registro do sinal atrial. Para ablação foram utilizados cateteres deflectíveis com ponta de 4mm, com ou sem controle de temperatura e geradores de RF, comercialmente disponíveis e selecionados, de acordo com a disponibilidade e preferência do operador.

Extra-estímulos atriais foram aplicados durante ciclos básicos de 600, 500 e, se necessário, 400ms, para demonstração de salto nodal e eco atrial, e para indução da TRN 9 . Considerou-se como salto nodal um aumento maior de $50 \mathrm{~ms}$ no intervalo AH com um decremento de $10 \mathrm{~ms}$ no intervalo de acoplamento do extra-estímulo atrial. Considerou-se como eco atrial, a despolarização atrial retrógrada através da via rápida nodal de um extra-estímulo atrial precoce conduzido em sentido anterógrado pela via lenta nodal ${ }^{8}$.

Para escolha do sítio de aplicação de RF, foram utilizados critérios anatômicos e eletrofisiológicos ${ }^{6,9-11}$. A porção do triângulo de Koch no septo interatrial, próxima ao anel tricuspídeo, foi dividida em três zonas anatômicas: zona 3 correspondendo ao terço posterior, em frente ao óstio do seio coronário; zona 1 - correspondendo ao terço anterior, próximo ao corpo do nó AV e zona 2 - correspondendo ao terço intermediário entre as zonas 1 e 3 (fig. 1). As aplicações foram realizadas de modo escalonado, iniciando-se na zona 3 , continuando-se na zona 2 e, se necessário, na zona 1 (critério anatômico). Nestes locais, procurou-se uma relação entre o eletrograma atrial e ventricular (relação A/V) $\leq 0,5$ (critério eletrofisiológico). O critério de fim de procedimento foi a não reindução da TRN com estimulação atrial programada após a infusão de isoproterenol realizada após as aplicações.

As aplicações de RF foram sempre realizadas em ritmo sinusal e eram imediatamente interrompidas caso fosse observado prolongamento do intervalo PR em ritmo sinusal, ou dissociação ventrículo-atrial durante taquicardia juncional. Nos últimos 70 pacientes, a monitorização da condução AV foi feita durante estimulação atrial rápida, com frequiência superior à taquicardia da taquicardia juncional mas inferior àquela que produzia bloqueio $\mathrm{AV}$ do $1^{\circ}$ grau ou fenômeno de Wenckenbach. O ciclo da estimulação durante taquicardia juncional era, portanto, estabelecido antes da aplicação do $1^{\circ}$ pulso de RF.

Definiu-se como modificação da via lenta nodal quando, ao final do procedimento, a TRN não era mais induzida, mesmo após a infusão de isoproterenol, mas persistia o salto nodal com ou sem eco atrial durante estimulação programada. Por sua vez, definiu-se como ablação da via lenta nodal aqueles procedimentos nos quais a arritmia não era mais induzida, após a infusão de isoproterenol, e não havia salto ou eco atrial durante estimulação atrial programada (fig. 2).

Nos casos em que ocorreu BAVT indesejado, foram avaliadas a frequiência da taquicardia juncional durante a aplicação de RF, a relação entre o eletrograma atrial e o ventricular no local da aplicação e a diferença entre o eletrograma atrial registrado no local da aplicação e o registrado no cateter em posição de His. Estes sinais já haviam sido previamente publicados como possíveis preditores do risco de BAVT durante a ablação de TRN ${ }^{15-18}$.

O seguimento clínico foi feito através de correspondência (questionário enviado aos pacientes) ou de contato telefônico a cada três meses após a ablação.

As variáveis são apresentadas como média e desvio padrão e os grupos foram comparados através do teste exato de Fischer. Considerou-se $\mathrm{p}<0,05$ como valor de significância estatística.

\section{Resultados}

No fim do procedimento, observaram-se completa eliminação da via lenta (grupo ablação) em 98 (78\%) pacientes

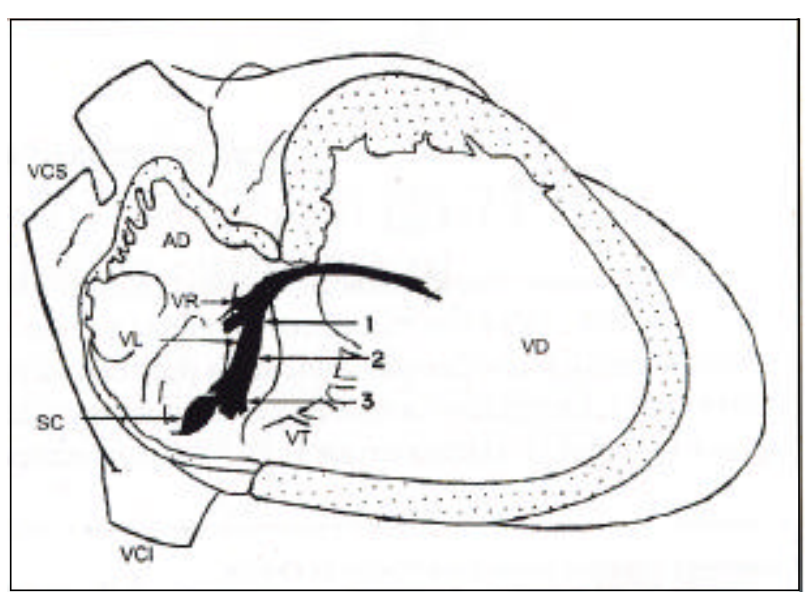

Fig. 1 - Zonas de ablação da via lenta - Esquema anatômico do coração visto em projeção oblíqua anterior direita. AD- átrio direito; VD- ventrículo direito; VT- valva tricúspide óstio; SC- do seio coronário; VCS- veia cava superior; VCI- veia cava inferior; VR- via rápida do nó AV; VL- via lenta do nó AV. 


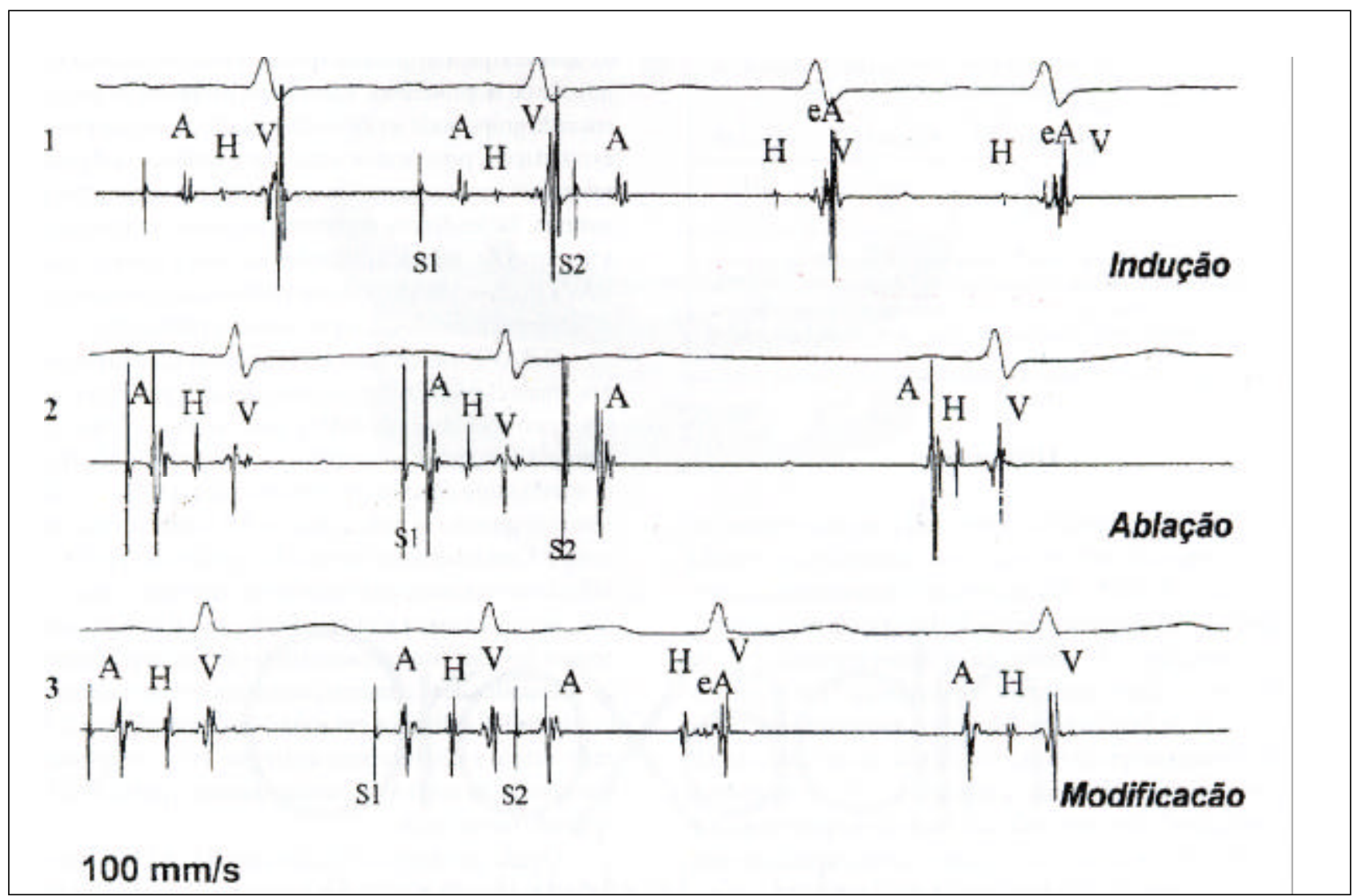

Fig. 2 - Traçados eletrofisiológicos obtidos durante indução(1), modificação (2) e ablação da via lenta nodal (3). A derivação eletrocardiográfica é DII. A- deflexão atrial; H- deflexão do feixe de His; V- deflexão ventricular; e A- eco atrial; S1, S2- artefatos dos estímulos atriais do ciclo básico e do extra-estímulo, respectivamente. Registros a 100mm/s.

e persistência de salto nodal com ou sem eco atrial (grupo modificação) em 28 (22\%) pacientes. Exemplos ilustrativos são apresentados na figura 2. Em ambos os grupos, a TRN não foi reinduzida. Não houve diferenças significativas entre os grupos estudados com relação à idade, sexo, número e local das aplicações de RF (tab. I).

Não foi observada diferença estatisticamente significativa com relação às recorrências de TRN entre os grupos estudados (tab. II). Houve recorrência em 15 (11\%) pacientes. A recorrência tendeu a ser maior no grupo modificação em relação ao grupo ablação (9\% vs 21\%), mas não houve significância estatística entre os resultados obtidos $(\mathrm{p}=0,09)$. Entre os pacientes com recorrência, não houve diferença em relação ao sexo, idade, número de aplicações e local de aplicação de RF com sucesso, que foi realizada exclusivamente na zona $3 \mathrm{em} 24$ (19\%) dos pacientes, na zona $2 \mathrm{em} 88(70 \%)$, e até a zona 1 em 14(11\%).

\begin{tabular}{|lccc|}
\hline \multicolumn{4}{|c|}{ Tabela I - Características clínicas dos grupos } \\
\hline & Ablação & Modificação & $\mathrm{p}$ \\
\hline Pacientes & 98 & 28 & - \\
Idade & 45 & 40 & 0,20 \\
Sexo feminino & $74 \%$ & $60 \%$ & - \\
Aplicação & 8 & 9 & 0,31 \\
Seguimento & 19 & 20 & 0,83 \\
\hline
\end{tabular}

Ocorreu indução acidental de BAVT em três dos 14 $(2,4 \%)$ pacientes, nos quais as aplicações foram realizadas na zona 1. Na análise desses casos, observou-se que os sinais eletrofisiológicos antes e durante a aplicação de RF, como a freqüência da taquicardia juncional, relação entre eletrograma atrial e ventricular no local da aplicação e a diferença entre o eletrograma atrial registrado no local da aplicação e o registrado no cateter em posição de His não foram diferentes das aplicações na zona 1 , que não resultaram em BAVT inadvertido (tab. III). Estes pacientes receberam marcapasso definitivo. As aplicações que resultaram em BAVT nos três pacientes foram, respectivamente, a $6^{\mathrm{a}}, 8^{\mathrm{a}} \mathrm{e}$ $16^{\mathrm{a}}$ aplicações. Nos três casos, aplicações anteriores nas zonas 3 e 2 não foram suficientes para suprimir a indução da TRN e o cateter foi, então, posicionado em zona 1.

O seguimento foi de $19 \pm 13$ meses no grupo ablação e $20 \pm 12$ meses no grupo modificação $(p=0,83)$.

\begin{tabular}{|lcccc|}
\hline \multicolumn{4}{|c|}{ Tabela II - Resultados } \\
\hline Grupo & $\mathrm{N}$ & Recorrência & $\mathrm{p}^{*}$ \\
\hline Ablação & 98 & & 9 & $(9 \%)$ \\
Modificação & 28 & 6 & $(21 \%)$ & 0,09 \\
\hline * teste exato de Fischer. \\
\hline
\end{tabular}




\begin{tabular}{|c|c|c|c|}
\hline & Frequêencia da TJ & Relação AV & $A(H)-(A B)$ \\
\hline Com BAVT* & 137 & 0,16 & 11 \\
\hline Sem BAVT* & 149 & 0,64 & 17 \\
\hline \multicolumn{4}{|c|}{$\begin{array}{l}\text { *p- não significativa; freqüência da TJ- frequiência cardíaca média da taquicardia } \\
\text { juncional durante a aplicação de radiofrequiência; relação } \mathrm{AV} \text { - relação A/V no } \\
\text { local da aplicação de sucesso; } \mathrm{A}(\mathrm{H}) \text { - } \mathrm{A}(\mathrm{AB}) \text { - diferença em ms entre a deflexão } \\
\text { atrial registrada no cateter posicionado em região de His e a deflexão atrial no } \\
\text { local da ablação. }\end{array}$} \\
\hline
\end{tabular}

\section{Discussão}

Não há um consenso sobre qual o melhor critério de fim de procedimento em pacientes submetidos à ablação por cateter de TRN. Alguns estudos demonstraram a necessidade da completa eliminação da via lenta para se evitar a recorrência ${ }^{4,5}$. A maioria dos estudos realizados ${ }^{1,4-8,12}$, entretanto, sugere que a não reindução da TRN é critério suficiente de fim de procedimento e que esses resultados independem de ter sido a escolha do sítio de aplicação de RF feita através de critérios anatômicos ${ }^{4,5,7,8,12}$ e/ou eletrofisiológicos ${ }^{6}$. Por outro lado, a infusão de isoproterenol foi considerada por esses investigadores como importante para determinar a possibilidade de recorrência, sugerindo, inclusive, que a não utilização rotineira de isoproterenol possa explicar os resultados conflitantes obtidos em outros estudos ${ }^{2}$.

Nossos resultados sugerem que a recorrência de TRN não depende do critério utilizado como final de procedimen- to, uma vez que a arritmia não pode ser mais reinduzida após a infusão de isoproterenol. Entretanto, o número de pacientes em cada grupo pode ter dificultado a aplicação dos métodos estatísticos, pois houve uma clara tendência de maior recorrência nos pacientes em que o salto e/ou o eco atrial persistiram. Tal tendência, entretanto, ao nosso ver, não justifica a tentativa de realizar aplicações na zona 1 onde o risco de BAVT inadvertido não pôde ser estabelecido, de acordo com critérios eletrofisiológicos previamente publicados.

OBAVT inadvertido ocorreu em três $(2,4 \%)$ pacientes e os sinais eletrofisiológicos considerados por alguns autores como preditores de BAVT inadvertido ${ }^{15-18}$ não foram úteis em diferenciar aplicações que causaram ou não BAVT. É interessante destacar, entretanto, que o BAVT ocorreu somente quando as aplicações de RF foram realizadas na zona 1. Com base nestes resultados, podemos sugerir que a ablação da via lenta seja tentada somente nas zonas $2 \mathrm{e} 3 \mathrm{e}$ que, nestes casos, a não reindução de TRN com isoproterenol deva ser considerada critério de fim de procedimento. A tentativa de se eliminar completamente a via lenta com pulsos de RF aplicados em zona 1 pode resultar em BAVT inadvertido e não há como distinguir entre as aplicações que resultarão em BAVT ou não, quando o pulso de RF for aplicado nessa região.

Desde que após a ablação com RF, a TRN não seja induzida através de estimulação programada após a infusão de isoproterenol, a taxa de recorrência não é diferente nos casos em que houve modificação da via lenta e que persistem com salto nodal e eco atrial, em comparação àqueles em que foi obtida a ablação da via lenta.

\section{Referências}

1. Haissaguerre M, Fischer B, Marcus F, Clementy J - Role of catheter ablation for treatment of supraventricular tachyarrithmias. In: Willian J Mandell - Cardiac Arrhytmias - $3^{\text {rd }}$ ed. 1995: 943-9.

2. Hummel J, Strickberger S, Williamson B et al - Effect of residual slow pathway function on the time course of recurrences of atrioventricular reentrant tachycardia after radiofrequency ablation of the slow pathway. Am J Cardiol 1995; 75: 628-30.

3. Chen $\mathrm{S}, \mathrm{Wu} \mathrm{T}$, Chiang $\mathrm{C}$ et al - Recurrent taquicardia after selective ablation of slow pathway in patients with atrioventricular nodal reentrant tachycardia. Am J Cardiol 1995; 76: 131-7.

4. Li H, Klein G, Stites Het al - Elimination of slow pathway conduction: An accurate indicator of clinical success after radiofrequency atrioventricular node modification. J Am Coll Cardiol 1993; 22: 1849-53.

5. Baker J, Plumb V, Epstein A, Kay G - Predictors of recurrent atrioventricular nodal reentry after selective slow pathway ablation. Am J Cardiol 1994; 73: 765-9.

6. Manolis A, Wang P, Estes III M - Radiofrequency ablation of slow pathway in patients with atrioventricular nodal reentrant tachycardia . Do arrhytmia recurrences correlate with persistent slow pathway conduction or site of succesful ablation? Circulation 1994; 90: 2815-9.

7. Wu D, Yeh S, Wang C, Wen M,Lin F - A simple technique for selective radiofrequency ablation of the slow pathway in atrioventricular node reentrant tachycardia. J Am Coll Cardiol 1993; 21: 1612-21.

8. Lindsay B, Chung M, Gamache M et al - Therapeutic end points for the treatment of atrioventricular node reentrant tachycardia by catheter-guided radiofrequency current. J Am Coll Cardiol 1993; 22: 733-40.

9. Strickberger S, DaoudE, Niebauer Met al-Effects of partial and complete ablation of the slow pathway on fast pathway properties in patients with atrioventricular nodal reentrant tachycardia. J Cardiovascr Electrophysiol 1994; 5: 645-9.
10. Tondo C, Bella P, Carbucicchio C, Riva S - Persistence of single echo beat inducibility after selective ablation of the slow pathway in patients with atrioventricular nodal reentrant tachycardia: relationship to the functional properties of the atrioventricular node and clinical implications. J Cardiovasc Electrophysiol 1996; 7: 689-96.

11. Josephson ME - Supraventricular Tachycardias. Clinical Cardiac Electrophysiology. Techniques and Interpretations. $2^{\text {nd }}$ ed.; Chapter 9: $\quad$ 181-274.

12. Jackman W, Bekman K, McClelland J et al - Treatment of supraventricular tachycardia due to atrioventricular nodal reentry by radiofrequency catheter ablation of slow pathway conduction. N Eng J Med 1992; 327: 313-18.

13. Haissaguerre M, Gaita F, Fischer B et al - Elimination of atrioventricular reentrant tachycardia using discret slow potentials to guide aplication of radiofrequency energy. Circulation 1992; 85: 2162-75.

14. Jazayeri M, Hempe S, Sra J et al - Selective transcatheter ablation of the fast and slow pathways using radiofrequency energy in patients with atrioventricular nodal reentrant tachycardia. Circulation 1992; 85: 1318-28.

15. Hintringer F, Hartikainen J, Davies W et al - Prediction of atrioventricular block during radiofrequency ablation of the slow pathway of the atrioventricular node. Circulation 1995; 92: 3490-6.

16. Jentzer J, Goyial R, Willianson B et al - Analysis of junctional ectopy during radiofrequency ablation of the slow pathway in patients with atrioventricular nodal reentrant tachycardia. Circulation 1994; 90: 2820-6.

17. Thakur R, Klein G, Yee R, Stites H - Junctional tachycardia: A useful marker during radiofrequency ablation for atrioventricular node reentrant tachycardia. $\mathrm{J}$ Am Coll Cardiol 1993; 22: 1706-10.

18. Whaten M, Natale A, Wolfe K, Yee R, Newmann D, Klein G - An anatomically guided approach to atrioventricular node slow pathway ablation. Am J Cardiol 1992; 70: 886-9. 